\title{
Puerarin transport across a Calu-3 cell monolayer - an in vitro model of nasal mucosa permeability and the influence of paeoniflorin and menthol
}

This article was published in the following Dove Press journal:

Drug Design, Development and Therapy

8 July 2016

Number of times this article has been viewed

\author{
Lin Zhang \\ Shou-Ying Du \\ Yang Lu \\ Chang Liu \\ Zhi-Hao Tian \\ Chang Yang \\ Hui-Chao Wu \\ Zhen Wang
}

School of Chinese Materia Medica, Beijing University of Chinese

Medicine, Chaoyang District, Beijing,

People's Republic of China
Correspondence: Shou-Ying Du; Yang Lu School of Chinese Materia Medica, Beijing University of Chinese Medicine, No 6, Zhonghuan South Road, Wangjing, Chaoyang District, Beijing I00I02,

People's Republic of China

Tel +861084738615

Fax +86101084738611

Email dushouying607816@163.com; landocean28@163.com

\begin{abstract}
Nasal administration is a high-potential delivery system, particularly because it can provide a pathway from the nose to the brain. The objective of this research is to characterize puerarin transport across a Calu-3 cell monolayer used as a model of the nasal mucosa and to evaluate the influence of puerarin in combination with paeoniflorin and menthol to explore the enhanced mechanism of the permeability at the cell level. The apparent permeability coefficients $\left(P_{\text {app }}\right)$ of puerarin bidirectional transport were both $<1.5 \times 10^{-6} \mathrm{~cm} / \mathrm{s}$, and the efflux ratio was $<1.5$, indicating that puerarin alone exhibited poor absorption and that its transport primarily occurred by passive diffusion through the cell monolayer. When puerarin was coadministered with paeoniflorin, the $P_{\text {app }}$ was not changed $(P>0.05)$. However, the addition of menthol significantly $(P<0.05)$ improved the $P_{\text {app }}$ of puerarin in both directions. Moreover, based on immunofluorescence experiments and transepithelial electrical resistance measurements, the data indicated that the drug compatibility opened tight junctions and weakened the barrier capabilities of epithelial cells, thereby promoting the permeability of puerarin.
\end{abstract}

Keywords: puerarin, paeoniflorin, menthol, nasal administration, transport, tight junction

\section{Introduction}

Nasal delivery is a method of drug administration in which drugs are absorbed from the nasal mucosa to produce a local or systemic effect; this administration method has received extensive attention in recent years. ${ }^{1}$ By nasal administration, the drug can obtain a rapid absorption, a high bioavailability, excellent effectiveness, and brain targeting. ${ }^{2,3}$ Particularly due to the pathway from the nose to the brain, an important physiological feature of the nose, significant efforts have been made over the past 10 years to develop nasal delivery systems that can potentiate the delivery of drugs to the central nervous system (CNS). ${ }^{4,5}$ Because of its functions of filtering and homeostatic maintenance, the nasal mucosa forms a nose-brain barrier ${ }^{6}$ to limit the transmission and bioavailability of the drugs to the CNS, which poses a challenge to nasal administration. To accurately elucidate the mechanisms underlying drug absorption and to determine how to increase drug permeability after nasal administration, various methods have been employed both in vivo and in vitro. Of these methods, in vitro cell culture models that accurately simulate the physiological properties of transport and permeation through nasal epithelial cells at the cellular level have become widely recognized. ${ }^{7,8}$ These cell culture models are divided into two types: real nasal mucosa models based on primary cultures of nasal epithelial cells from various species and surrogate nasal mucosa models, which generally use Calu-3 cells, RPMI2650 cells, 
or other appropriate cell lines. ${ }^{9-14}$ In this research, we chose to use Calu-3 cells to stimulate the nasal mucosa barrier function in vitro because Calu- 3 cells have been previously used to study nasal drug absorption. ${ }^{15-17}$

Tongqiaosanyu, a traditional Chinese medicine used to treat acute cerebral stroke, mainly consists of the kudzu root (Radix Puerariae Lobatae), white peony root (Radix Paeoniae Alba), and menthol (Herba Menthae). In traditional Chinese medicine, kudzu roots ${ }^{18-21}$ are usually used to treat cardiovascular disorders and ischemic stroke; puerarin (Figure 1A), the major isoflavone glycoside isolated from kudzu root, exhibits a therapeutic effect when prescribed to treat cerebral disease. ${ }^{22}$ White peony root is usually administered together with kudzu root to treat acute cerebral stroke in traditional Chinese medicine clinics. ${ }^{23-25}$ Pharmacodynamic screening has revealed that the effective constituent of white peony root is paeoniflorin (Figure 1B), ${ }^{26}$ a monoterpene glucoside with many therapeutic functions, such as antithrombotic activity, anti-inflammatory activity, enhancement of glucose uptake, and neuroprotective effects. ${ }^{27,28}$ However, because puerarin and paeoniflorin display low absorption and bioavailability when administered orally, their applications are restricted in clinical settings. Menthol (Figure 1C) is a major constituent of peppermint oil and has been confirmed to excite the CNS and enhance permeation. ${ }^{29-31}$ Our ultimate aim is to provide the full benefits of puerarin in Tongqiaosanyu for the treatment of certain brain diseases by developing a nasal drug delivery system that takes advantage of the compatibility of puerarin, paeoniflorin, and menthol.

Previous research examining this medication evaluated the effects of different administration routes and compatibility on its pharmacokinetic behavior in vivo. To study the pharmacokinetic behavior of puerarin in rats following different methods of administration, Tongqiaosanyu was administered to rats by caudal vein injection, nasal administration, and oral administration, and the concentration of the puerarin in mice plasma and brain was analyzed by reversed phase high-performance liquid chromatography (HPLC). The result showed that the area under the plasma concentration-time curve from zero to infinity $\left(\mathrm{AUC}_{0-\infty}\right)$ of caudal vein injection was $787.99 \pm 70.44 \mathrm{mg} \cdot \mathrm{min} \cdot \mathrm{L}^{-1} ; \mathrm{AUC}_{0-\infty}$ of nasal administration was $376.56 \pm 93.93 \mathrm{mg} \cdot \mathrm{min} \cdot \mathrm{L}^{-1}$; $\mathrm{AUC}_{0-\infty}$ of oral administration (the dose was ten times higher than that of caudal vein injection and nasal administration) was $491.18 \pm 110.64 \mathrm{mg} \cdot \mathrm{min} \cdot \mathrm{L}^{-1}$; the absolute bioavailability of puerarin was $47.78 \%$ by nasal administration; the brain targeting coefficient $(R e)$ was $132.25 \%$ by intranasal administration, and the brain drug targeting index was 2.70, which were significantly higher than those by the injections and oral administration. These results indicated that the bioavailability of puerarin by intranasal administration is significantly higher than that by oral administration and the brain drug targeting of puerarin by intranasal administration is higher than that by injection and oral administration, which shows the advantage on the medicine absorption into the brain by the intranasal administration. ${ }^{32-34}$ However, for these compounds, the transport across the nasal mucosa and the influence of compatibility on nasal epithelial cells remain unclear. Thus, Calu-3 cell culture was used in this study to simulate the nasal mucosa in vitro to elucidate the permeability of puerarin and the contribution of mutual compatibility to enhancing permeability. Additionally, further analysis at the cell level was performed to determine the drug actions on the barrier functions and the associated protein structures of the monolayer. These experiments will provide fundamental data for the future study of the complicated nose-brain pathway.
A

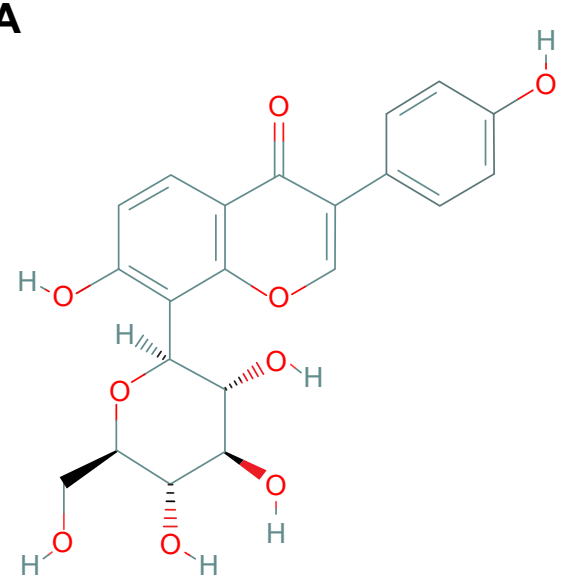

B

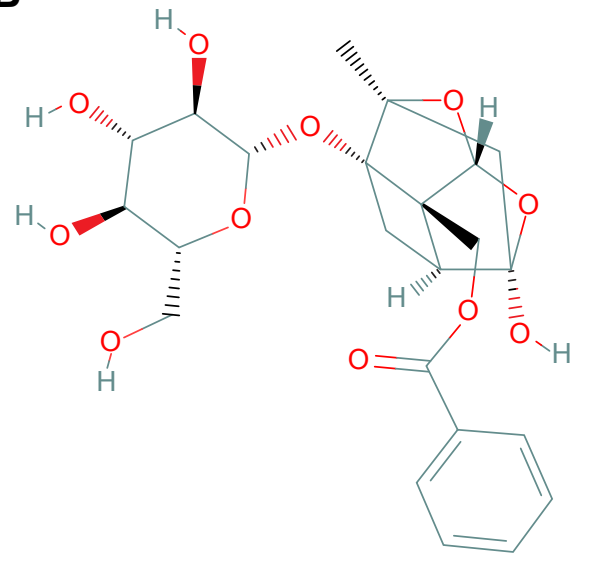

C

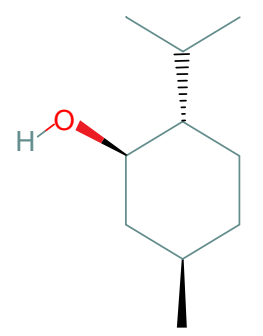

Figure I Chemical structures of (A) puerarin, (B) paeoniflorin, and (C) menthol. 


\section{Materials and methods Materials}

Puerarin, paeoniflorin, and menthol were obtained from the National Institute for the Control of Pharmaceutical and Biological Products (Beijing, People's Republic of China). Calu-3 cells were purchased from China Infrastructure of Cell Line Resources (Beijing, People's Republic of China). Polyethylene terephthalate (PET) cell culture inserts and 12-well plates (12 $\mathrm{mm}$ diameter, $0.4 \mu \mathrm{m}$ pore size) were purchased from Corning Incorporated (Corning, NY, USA). A rabbit anti-occludin antibody (ab31721) was obtained from Abcam Shanghai Co., Ltd (Shanghai, People's Republic of China). A mouse anti-claudin-1 antibody (2H10D10) was purchased from Thermo Fisher Scientific (Waltham, MA, USA). A tetramethylrhodamine isothiocyanate (red)-conjugated anti-rabbit immunoglobulin $\mathrm{G}(\mathrm{IgG})$ antibody was obtained from Beijing Zhongshan Golden Bridge Biotechnology Co. Ltd (Beijing, People's Republic of China). A fluorescein isothiocyanate (green)-conjugated anti-mouse IgG antibody was purchased from Kangwei Century Biotechnology Co. Ltd (Beijing, People's Republic of China). Acti-stain488 (green) fluorescent phalloidin was procured from Cytoskeleton Inc. (Denver, $\mathrm{CO}$, USA). In this research, all experimental protocols were approved by the Review Committee for the Use of Human or Animal Subjects of Beijing University of Chinese Medicine.

\section{Cell culture}

Calu-3 cells were purchased from the Institute of Basic Medical Sciences, Chinese Academy of Medical Sciences (Beijing, People's Republic of China) and were negative for the mycoplasma infection test. The cells were used at passage numbers 5-10 and were grown in minimum essential medium (Thermo Fisher Scientific) with $10 \%$ heat-inactivated fetal bovine serum (Thermo Fisher Scientific), $100 \mathrm{U} / \mathrm{mL}$ penicillin, and $100 \mu \mathrm{g} / \mathrm{mL}$ streptomycin in a humidified atmosphere with 5\% $\mathrm{CO}_{2}$ at $37^{\circ} \mathrm{C}$. Upon reaching $90 \%$ confluence, the cells were trypsinized using $0.25 \%$ trypsin and $0.1 \%$ ethylenediaminetetraacetic acid. Post-trypsinization, the Calu- 3 cell suspension $(0.5 \mathrm{~mL})$ was inoculated at a density of $5 \times 10^{5}$ cells $/ \mathrm{mL}$ on to $0.4 \mu \mathrm{m}$ pore, collagen-coated clear PET membranes in $12 \mathrm{~mm}$ Transwell chambers, and medium $(0.5 \mathrm{~mL})$ was added to the basolateral chamber. On day 5 , the medium on the apical surface was removed to produce air interface feeding conditions, and the transepithelial electrical resistance (TEER) values were measured by chopstick electrodes and an epithelial volt-ohm meter (EMD Millipore, Billerica, MA, USA) every other day when the medium in the basolateral chamber was replaced. After an additional 10 days, when the cells had reached maximum confluence on $\sim 100 \%$ of the permeable support membranes and the TEER values had increased to $>500 \Omega \mathrm{cm}^{2}$ under the conditions described earlier, the experiments were performed on polarized Calu-3 cell layers. For all the experiments, similar passage numbers were used.

\section{Cytotoxicity assays}

The levels of puerarin, paeoniflorin, and menthol that could interfere with the growth of Calu-3 cells were determined using the 3-(4,5-dimethylthiazol-2-yl)-2,5-diphenyltetrazolium bromide (MTT) dye assay. The cells were seeded onto 96-well microtiter plates with flat-bottomed wells in a total volume of $100 \mu \mathrm{L}$ of culture medium at a density of $1 \times 10^{5}$ cells $/ \mathrm{mL}$ and incubated in a humidified atmosphere with $5 \% \mathrm{CO}_{2}$ at $37^{\circ} \mathrm{C}$. After 48 hours, the medium was removed and replaced with fresh medium containing different compounds at various concentrations. After culturing for an additional 48 hours, $15 \mu \mathrm{L}$ of $5 \mathrm{mg} / \mathrm{mL}$ MTT in phosphate-buffered saline (PBS) was added to each well, and the mixtures were incubated at $37^{\circ} \mathrm{C}$ for 4 hours until purple deposits became visible. This assay measures the production of purple formazan, an MTT reaction product generated by mitochondrial dehydrogenase that indicates cell viability; in this reaction, formazan production is proportional to reductive activity. After the MTT solution was discarded, the colored reaction products were completely dissolved by the addition of $150 \mu \mathrm{L}$ of dimethyl sulfoxide, and the absorbance was measured at $490 \mathrm{~nm}$ using a Multiskan GO microplate reader (Thermo Fisher Scientific). The mean absorbance of five measurements for each compound was expressed as the percentage of the absorbance of the untreated control and plotted against the concentration of the compound.

Cell viability (percentage) from the MTT assay was calculated using the following equation:

$$
\text { Viability }(\%)=\frac{A_{\text {sample }}-A_{\text {blank }}}{A_{\text {control }}-A_{\text {blank }}} \times 100
$$

in which $A$ is the absorbance value.

\section{Evaluation of transport across Calu-3 cells}

To determine the permeability of puerarin and the effect of different drugs on its penetration, drug transport in cell monolayers was analyzed. First, Calu- 3 cells were seeded at a density of $5 \times 10^{5}$ cells $/ \mathrm{mL}$ on to the PET membranes in Transwell chambers and allowed to form monolayers 
(TEER $>500 \Omega \cdot \mathrm{cm}^{2}$ ). Before each experiment, the cells were washed three times with Hank's balanced salt solution (HBSS) and equilibrated for 30 minutes at $37^{\circ} \mathrm{C}$. The drug solution $(0.5 \mathrm{~mL})$ was added to the apical (A) side, and HBSS $(1.5 \mathrm{~mL})$ was added to the basolateral $(\mathrm{B})$ side to measure $\mathrm{A} \rightarrow \mathrm{B}$ transport. The cells were incubated at $37^{\circ} \mathrm{C}$ with shaking. Samples $(600 \mu \mathrm{L})$ were collected from the B side at 30 minutes, 60 minutes, 90 minutes, 120 minutes, 150 minutes, and 180 minutes. The amount of puerarin transported was measured with HPLC using a Hibar $\mathrm{C}_{18}$ column ( $\left.4 \times 200 \mathrm{~mm}^{2}, 5 \mu \mathrm{m}\right)$, and the samples were analyzed via UV detection $(\lambda=250 \mathrm{~nm})$. The mobile phase consisted of methanol and $1 \%$ acetate solution $(37: 63, \mathrm{v} / \mathrm{v})$ and was pumped at a flow rate of $1 \mathrm{~mL} / \mathrm{min}$, and the injected volume was $20 \mu \mathrm{L}$. Under these conditions, the retention time of puerarin was $\sim 6$ minutes. $\mathrm{B} \rightarrow \mathrm{A}$ transport was evaluated by adding $1.5 \mathrm{~mL}$ of drug solution to the $\mathrm{B}$ side and $0.5 \mathrm{~mL}$ of HBSS to the A side. Samples $(200 \mu \mathrm{L})$ were collected from the A side at the same time and measured with the same HPLC method used to assess $\mathrm{A} \rightarrow \mathrm{B}$ transport.

The apparent permeability coefficients $\left(P_{\text {app }}\right)$ for puerarin were calculated according to the following equation:

$$
P_{\text {app }}=\frac{(d Q / d t)}{C S}
$$

in which $d Q / d t$ is the apparent appearance rate of puerarin in the receiver side, which was calculated by linear regression of the amount of puerarin in the receiver chamber at different time points; $C$ is the puerarin concentration in the donor chamber; and $S$ is the surface area of the PET membrane of the Transwell chamber.

The efflux ratio (ER) was calculated according to the following equation:

$$
\mathrm{ER}=\frac{P_{\text {app }}(\mathrm{B} \rightarrow \mathrm{A})}{P_{\text {app }}(\mathrm{A} \rightarrow \mathrm{B})}
$$

\section{Measurement of cell TEER changes after exposure to different compounds}

In addition to determining the integrity of monolayers, the cell TEER values were measured to investigate changes in the intercellular compactness of the Calu-3 cells. After the cells had formed monolayers, the drug solution $(0.5 \mathrm{~mL})$ was added to the A side and $\operatorname{HBSS}(1.5 \mathrm{~mL})$ was added to the $\mathrm{B}$ side to simulate $\mathrm{A} \rightarrow \mathrm{B}$ transport. $\mathrm{B} \rightarrow \mathrm{A}$ transport was evaluated by adding the drug solution $(0.5 \mathrm{~mL})$ to the $\mathrm{B}$ side and HBSS (1.5 mL) to the A side. The TEER values of the untreated cells and the cells treated with different drugs were determined at 30 minutes, 60 minutes, 90 minutes, 120 minutes, 150 minutes, and 180 minutes. The measured TEER before the experiment was set as $100 \%$, and all the other values were calculated relative to this value. Then, the relative TEER at each time point was compared with the control group value and statistically analyzed.

\section{Immunofluorescence microscopy}

For the immunocytochemical assessment of tight junction (TJ) proteins, cells were seeded at a density of $5 \times 10^{5}$ cells $/ \mathrm{mL}$ onto the PET membranes in Transwell chambers until they formed monolayers. Next, the cells were cultured with media containing different compounds for 3 hours. At the beginning of the experiment, the cells were fixed with cold $4 \%$ paraformaldehyde for 30 minutes. After a rinse in PBS, the cells were incubated with goat serum as a blocking buffer for 1 hour, and the samples were subsequently incubated with a polyclonal anti-occludin (1:100) or monoclonal anti-claudin-1 (1:100) primary antibody at $4{ }^{\circ} \mathrm{C}$ overnight. After being washed three times with PBS again, the cells were incubated with the secondary antibody, tetramethylrhodamine isothiocyanate (red)-conjugated anti-rabbit IgG or fluorescein isothiocyanate (green)-conjugated anti-mouse IgG. Some cells were stained only with Acti-stain 488 (green) fluorescent phalloidin (1:150) at room temperature for 30 minutes. DAPI (Solarbio, Beijing Solarbio Science and Technology Co., Ltd, Beijing, People's Republic of China) was used to counterstain cell nuclei. The membranes were carefully excised from Transwell inserts, mounted on a glass slide with $80 \%$ glycerol, and covered with a $15 \mathrm{~mm}$ coated glass coverslip. The morphology and fluorescence of TJ proteins were visualized using an inverted fluorescence microscope equipped with an appropriate filter (Olympus Corporation, Tokyo, Japan) and a laser-scanning confocal microscope, and images were obtained using the accompanying analysis software. The settings for image collection were identical, and the average optical density (AOD) of the images was calculated using ImageJ software.

The immunofluorescence images were semiquantitatively determined using Image J software according to the following equation:

$$
\mathrm{AOD}=\frac{\text { IntDen }}{\text { Area }}
$$

in which IntDen is the integrated optical density in the image and Area is the region of fluorescence in the image. 
The AOD percentage corresponding to the TJ protein was calculated using the following equation:

$$
\text { Relative AOD }(\%)=\frac{\mathrm{AOD}_{\text {sample }}}{\mathrm{AOD}_{\text {control }}} \times 100
$$

\section{Data analysis and statistics}

Each set of results shown is representative of three separate experiments. The results are given as the mean $\pm \mathrm{SD}$. The data were analyzed with one-way analysis of variance followed by the Dunnett's test to compare differences between multiple groups and the control group (using SPSS 17.0 statistical software; SPSS Inc., Chicago, IL, USA). Significance was set at $P<0.05$.

\section{Results}

\section{Cytotoxicity of compounds in Calu-3 cells}

The cytotoxicity results are shown in Figure 2. The puerarin, paeoniflorin, and menthol groups showed no cytotoxicity in the concentration ranges of $0-350 \mu \mathrm{g} / \mathrm{mL}, 0-250 \mu \mathrm{g} / \mathrm{mL}$, and $0-60 \mu \mathrm{g} / \mathrm{mL}$, respectively (Figure $2 \mathrm{~A}-\mathrm{C}$ ). The puerarin plus
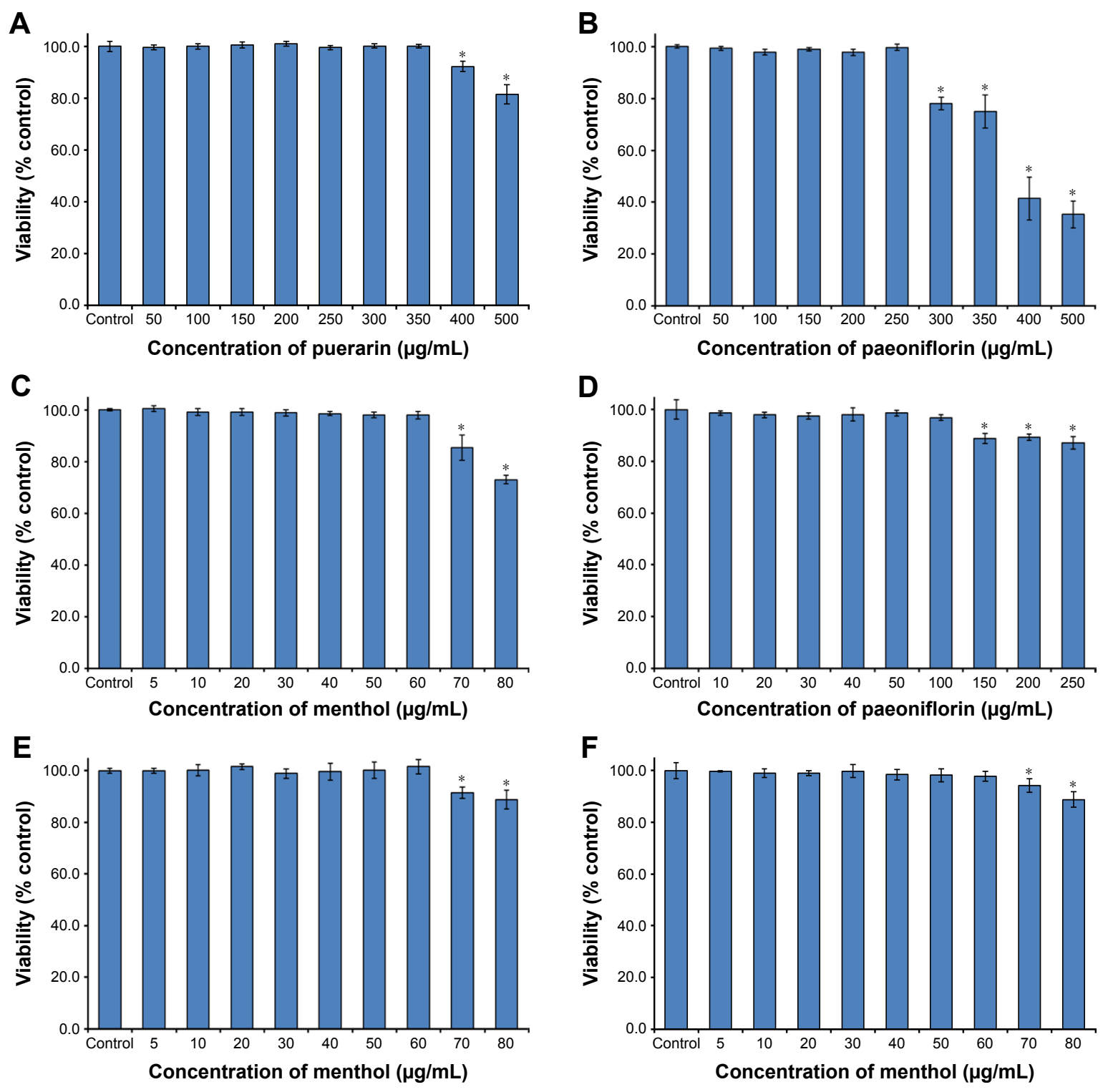

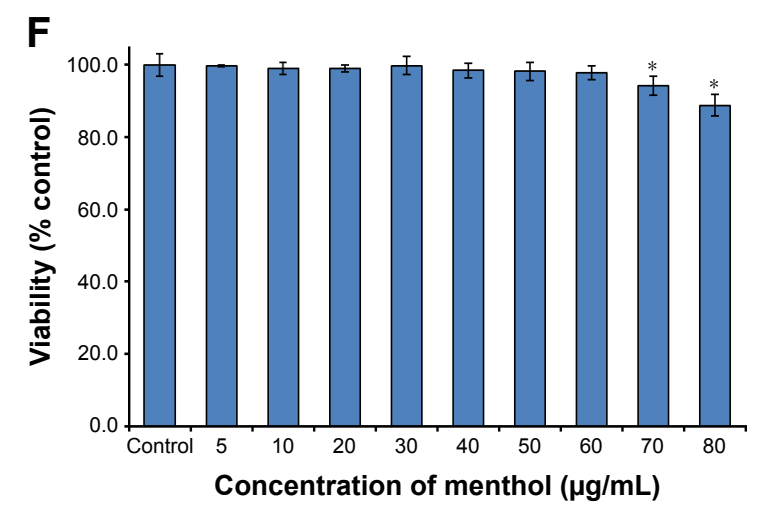

Figure 2 Cytotoxicity of puerarin, paeoniflorin, menthol, and their combinations as assessed by MTT test in Calu-3 cells for 24 hours.

Notes: (A) Cytotoxicity of puerarin, (B) cytotoxicity of paeoniflorin, (C) cytotoxicity of menthol, (D) cytotoxicity of puerarin combined with paeoniflorin, (E) cytotoxicity of puerarin combined with menthol, and (F) cytotoxicity of puerarin combined with paeoniflorin and menthol. Data expressed as mean \pm SD ( $n=5)$. $* P<0.05$ compared with the control group.

Abbreviations: MTT, 3-(4,5-dimethylthiazol-2-yl)-2,5-diphenyltetrazolium bromide; SD, standard deviation. 
paeoniflorin group (puerarin:paeoniflorin, 1:0.4, w/w) showed no cytotoxicity at concentrations of $0-100 \mu \mathrm{g} / \mathrm{mL}$, which was graphed against paeoniflorin concentration (Figure 2D). The puerarin plus menthol group (puerarin:menthol, 1:0.5, w/w) showed no cytotoxicity at concentrations of $0-60 \mu \mathrm{g} / \mathrm{mL}$, which was graphed in terms of the menthol concentration (Figure 2E). The puerarin plus paeoniflorin and menthol group (puerarin:paeoniflorin:menthol, 1:0.4:0.5, w/w/w) showed no cytotoxicity at concentrations of $0-60 \mu \mathrm{g} / \mathrm{mL}$, which was graphed against the menthol concentration (Figure $2 \mathrm{~F}$ ).

\section{Transport of puerarin}

Puerarin transport across Calu-3 cells was studied at three concentrations: $30 \mu \mathrm{g} / \mathrm{mL}, 60 \mu \mathrm{g} / \mathrm{mL}$, and $120 \mu \mathrm{g} / \mathrm{mL}$. The results are shown in Table 1 . The $P_{\text {app }}(\mathrm{A} \rightarrow \mathrm{B})$ of puerarin was between $1.220 \times 10^{-6} \mathrm{~cm} / \mathrm{s}$ and $1.238 \times 10^{-6} \mathrm{~cm} / \mathrm{s}$, whereas the $P_{\text {app }}(\mathrm{B} \rightarrow \mathrm{A})$ of puerarin was between $1.174 \times 10^{-6} \mathrm{~cm} / \mathrm{s}$ and $1.266 \times 10^{-6} \mathrm{~cm} / \mathrm{s}$ in Calu-3 cells. The fluxes of puerarin from the $A$ side to the $B$ side showed no significant difference compared with the fluxes from the B side to the A side $(P>0.05)$. The ER for each puerarin concentration was $\sim 1$.

The effects of different drug interactions on puerarin transport in Calu-3 cells are shown in Table 2. The effects of paeoniflorin were studied at $12 \mu \mathrm{g} / \mathrm{mL}, 24 \mu \mathrm{g} / \mathrm{mL}$, and $48 \mu \mathrm{g} / \mathrm{mL}$ concentrations in the presence of $60 \mu \mathrm{g} / \mathrm{mL}$ puerarin. In combination with different concentrations of paeoniflorin, the $P_{\text {app }}(\mathrm{A} \rightarrow \mathrm{B}$ and $\mathrm{B} \rightarrow \mathrm{A})$ values did not differ significantly from those of the control group $(P>0.05)$, indicating that paeoniflorin could not alter the puerarin flux. The effects of menthol were studied at $15 \mu \mathrm{g} / \mathrm{mL}, 30 \mu \mathrm{g} / \mathrm{mL}$, and $60 \mu \mathrm{g} / \mathrm{mL}$ concentrations in the presence of $60 \mu \mathrm{g} / \mathrm{mL}$ puerarin. Menthol significantly increased the puerarin flux in both directions in a concentration-dependent manner $(30 \mu \mathrm{g} / \mathrm{mL}$ and $60 \mu \mathrm{g} / \mathrm{mL}$ in Calu-3 cells, $P<0.05)$. The effects of both paeoniflorin and menthol were studied at the aforementioned concentrations in the presence of $60 \mu \mathrm{g} / \mathrm{mL}$ puerarin. Paeoniflorin and menthol together also significantly increased the puerarin flux in both directions in a concentration-dependent

Table I Transport of increasing puerarin concentrations across Calu-3 cell monolayers

\begin{tabular}{llll}
\hline $\begin{array}{l}\text { Condition } \\
(\mu \mathrm{g} / \mathrm{mL})\end{array}$ & $\begin{array}{l}\boldsymbol{P}_{\text {app }}(\mathbf{A} \rightarrow \mathbf{B}) \\
\left(\times 10^{-6} \mathbf{c m} / \mathbf{s}\right)\end{array}$ & $\begin{array}{l}\boldsymbol{P}_{\text {app }}(\mathbf{B} \rightarrow \mathbf{A}) \\
\left(\times 10^{-6} \mathbf{c m} / \mathbf{s}\right)\end{array}$ & $\begin{array}{l}\text { ER }(\mathbf{B} \rightarrow \mathbf{A} / \\
\mathbf{A} \rightarrow \mathbf{B})\end{array}$ \\
\hline 30 & $1.220 \pm 0.027$ & $1.174 \pm 0.053$ & $0.963 \pm 0.065$ \\
60 & $1.226 \pm 0.039$ & $1.240 \pm 0.055$ & $1.012 \pm 0.032$ \\
120 & $1.238 \pm 0.053$ & $1.266 \pm 0.104$ & $1.023 \pm 0.070$ \\
\hline
\end{tabular}

Notes: $P_{\text {app }}$, permeability; A, apical side; B, basolateral side. Values shown as mean \pm $\operatorname{SD}(n=3)$.

Abbreviations: ER, efflux ratio; s, seconds; SD, standard deviation.
Table 2 Effects of paeoniflorin $(\mathrm{Pa})$ and menthol $(\mathrm{Me})$ on puerarin $(\mathrm{Pu})$ transport in Calu-3 cells

\begin{tabular}{|c|c|c|c|}
\hline $\begin{array}{l}\text { Condition } \\
(\mu \mathrm{g} / \mathrm{mL})\end{array}$ & $\begin{array}{l}P_{\text {app }}(A \rightarrow B) \\
\left(\times 10^{-6} \mathrm{~cm} / \mathrm{s}\right)\end{array}$ & $\begin{array}{l}P_{\text {app }}(B \rightarrow A) \\
\left(\times 10^{-6} \mathrm{~cm} / \mathrm{s}\right)\end{array}$ & $\begin{array}{l}E R(B \rightarrow A / \\
A \rightarrow B)\end{array}$ \\
\hline Pu (60) (control) & $1.226 \pm 0.039$ & $1.240 \pm 0.055$ & $1.012 \pm 0.032$ \\
\hline$+\mathrm{Pa}(12)$ & $1.240 \pm 0.027$ & $1.242 \pm 0.037$ & $1.002 \pm 0.039$ \\
\hline$+\mathrm{Pa}(24)$ & $1.233 \pm 0.054$ & $1.254 \pm 0.032$ & $1.018 \pm 0.065$ \\
\hline$+\mathrm{Pa}(48)$ & $1.229 \pm 0.025$ & $1.289 \pm 0.035$ & $1.049 \pm 0.027$ \\
\hline$+\mathrm{Me}(15)$ & $1.285 \pm 0.084$ & $1.363 \pm 0.083$ & $1.065 \pm 0.116$ \\
\hline$+\operatorname{Me}(30)$ & $1.639 \pm 0.058 *$ & $1.803 \pm 0.111 *$ & $1.100 \pm 0.060$ \\
\hline$+\operatorname{Me}(60)$ & $1.788 \pm 0.087^{*}$ & $2.067 \pm 0.15 I^{*}$ & $1.157 \pm 0.088$ \\
\hline$+\mathrm{Pa}(12)+\mathrm{Me}(15)$ & $1.305 \pm 0.150$ & $1.288 \pm 0.067$ & $0.999 \pm 0.150$ \\
\hline$+\mathrm{Pa}(24)+\mathrm{Me}(30)$ & $1.559 \pm 0.143 *$ & $1.910 \pm 0.254^{*}$ & $1.228 \pm 0.157$ \\
\hline$+\mathrm{Pa}(48)+\mathrm{Me}(60)$ & $1.957 \pm 0.067 *$ & $2.136 \pm 0.130 *$ & $1.091 \pm 0.048$ \\
\hline
\end{tabular}

Notes: $P_{\text {app }}$, permeability; A, apical side; B, basolateral side. Values shown as mean \pm SD $(n=3)$. Differs from Pu $(60 \mu \mathrm{g} / \mathrm{mL}): * P<0.05$.

Abbreviations: ER, efflux ratio; s, seconds; SD, standard deviation.

manner $(24 \mu \mathrm{g} / \mathrm{mL}$ paeoniflorin and $30 \mu \mathrm{g} / \mathrm{mL}$ menthol or $48 \mu \mathrm{g} / \mathrm{mL}$ paeoniflorin and $60 \mu \mathrm{g} / \mathrm{mL}$ menthol, $P<0.05)$.

\section{Changes in TEER after treatment with various compounds}

TEER was measured in barrier function assays to investigate changes to the TJs in Calu-3 cells after exposure to puerarin, paeoniflorin, and menthol. The results are shown in Figure 3. In Calu-3 cells, the puerarin group $(30 \mu \mathrm{g} / \mathrm{mL}, 60 \mu \mathrm{g} / \mathrm{mL}$, and $120 \mu \mathrm{g} / \mathrm{mL}$ puerarin) (Figure $3 \mathrm{~A})$ and the PP group $(60 \mu \mathrm{g} / \mathrm{mL}$ puerarin combined with $12 \mu \mathrm{g} / \mathrm{mL}, 24 \mu \mathrm{g} / \mathrm{mL}$, and $48 \mu \mathrm{g} / \mathrm{mL}$ paeoniflorin) (Figure $3 \mathrm{~B}$ ) showed no difference from the control group regarding the trend of TEER change. In the PM group (60 $\mu \mathrm{g} / \mathrm{mL}$ puerarin plus $15 \mu \mathrm{g} / \mathrm{mL}, 30 \mu \mathrm{g} / \mathrm{mL}$, and $60 \mu \mathrm{g} / \mathrm{mL}$ menthol) (Figure 3C) and the PPM group (60 $\mu \mathrm{g} / \mathrm{mL}$ puerarin plus $12 \mu \mathrm{g} / \mathrm{mL}, 24 \mu \mathrm{g} / \mathrm{mL}$, and $48 \mu \mathrm{g} / \mathrm{mL}$ paeoniflorin and $15 \mu \mathrm{g} / \mathrm{mL}, 30 \mu \mathrm{g} / \mathrm{mL}$, and $60 \mu \mathrm{g} / \mathrm{mL}$ menthol) (Figure 3D), statistical analysis indicated that TEER began to decrease at 60 minutes $(P<0.05)$ for both the $\mathrm{A} \rightarrow \mathrm{B}$ and $\mathrm{B} \rightarrow \mathrm{A}$ transport processes. The decreased TEER, which resulted from compatibility with menthol, showed concentration-dependent behavior in the studied range.

\section{Effect of the compounds on TJ proteins}

To investigate the effect of the compounds on TJ proteins in Calu-3 cells, immunohistochemistry was performed to detect occludin, claudin-1, and F-actin, and the AOD of the images was measured. The images are shown in Figure 4, and the results calculated from the AOD values are shown in Figure 5. Three TJ proteins were clearly observed in Calu-3 cells. Occludin was stained red, claudin-1 and F-actin were stained green, and the nucleus was stained blue. After treatment with 

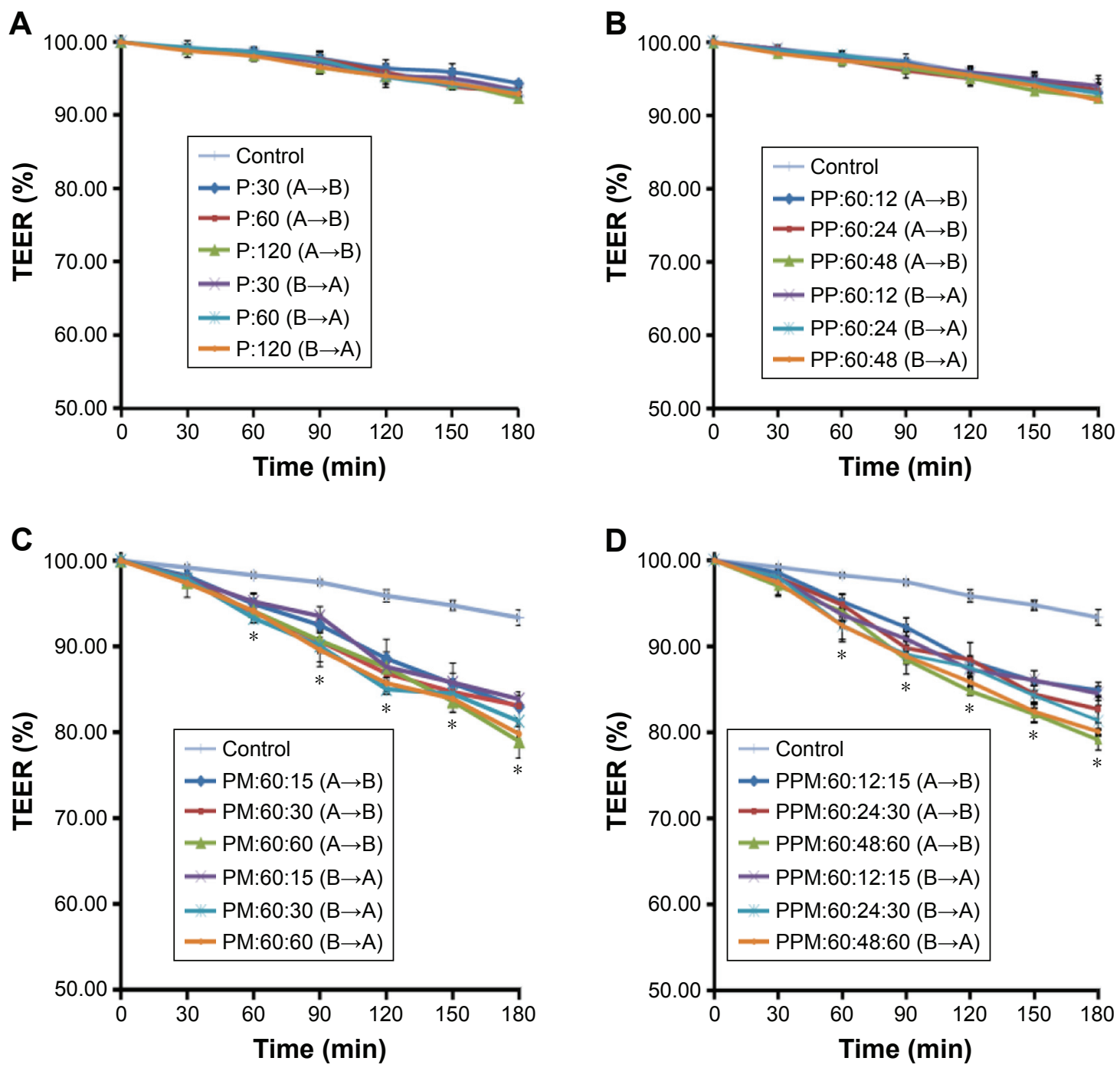

Figure 3 The trend of TEER change in $\mathrm{A} \rightarrow \mathrm{B}$ and $\mathrm{B} \rightarrow \mathrm{A}$ transport in Calu-3 cells after treatment with puerarin, either alone or in combination with paeoniflorin and menthol. Notes: (A) Exposure to different concentrations of puerarin, and no significant TEER change was observed compared with the control group. (B) Exposure to a specific amount of puerarin combined with different concentrations of paeoniflorin, and TEER also showed no significant change. (C) Exposure to a specific amount of puerarin combined with different concentrations of menthol, and TEER decreased significantly compared with the control group. (D) Exposure to a specific amount of puerarin combined with different concentrations of paeoniflorin and menthol, and TEER tended to decrease. The TEER at each time point is represented by the mean \pm SD ( $n=3$ ). Significant difference from TEER of the cells without treatment: $* P<0.05$.

Abbreviations: TEER, transepithelial electrical resistance; min, minutes; SD, standard deviation; P, puerarin; PP, puerarin and paeoniflorin; PM, puerarin and menthol; PPM; puerarin, paeoniflorin and menthol.

puerarin and paeoniflorin, the immunostaining results for the three types of TJ proteins were similar to the control group. However, when the cells were incubated with menthol, the fluorescence intensity was weaker than that of the control group. Moreover, as calculated by ImageJ software and analyzed with SPSS 17.0, the control, puerarin, and paeoniflorin groups exhibited similar AOD values, whereas the difference was statistically significant when the menthol group was compared with the control group $(P<0.05)$.

\section{Discussion}

In this study, we were able to show the characteristics of puerarin transport and the influence of paeoniflorin and menthol in a cell model. We also demonstrated that menthol altered the physiological activity of the monolayer, resulting in improved puerarin transport across Calu-3 cells. Calu-3 cells, which are derived from a human lung adenocarcinoma cell line, can form ion channels, and express the cystic fibrosis transmembrane conductance regulator protein, ${ }^{14,17}$ are regularly used to mimic the nasal mucosal tissue to investigate puerarin uptake and the effects of mutual drug compatibility. In terms of cytotoxicity, living cells can reduce MTT to violet formazan dye, whereas dead cells cannot. Thus, the viability of cells after exposure to different compounds may be evaluated by measuring MTT metabolism. The experimental results indicated that menthol was the most toxic, whereas puerarin 

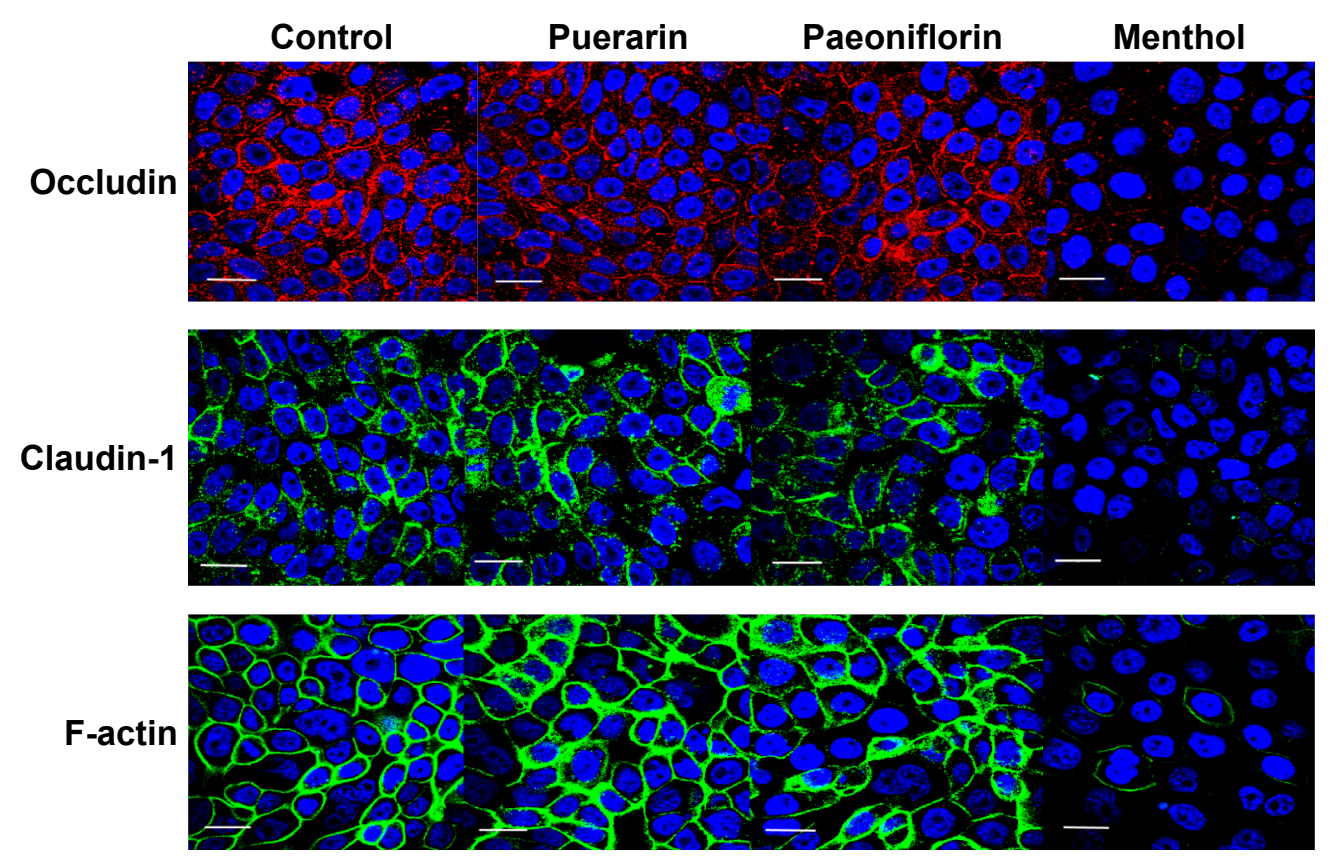

Figure 4 Effects of puerarin, paeoniflorin, and menthol on TJ proteins in Calu-3 cells.

Notes: Immunocytochemistry showed no differences in staining for the three types of TJ proteins in Calu- 3 cells treated with puerarin and paeoniflorin compared with the control group, whereas the three TJ proteins showed decreased staining after menthol treatment. The scale bar corresponds to $15 \mu \mathrm{m}$.

Abbreviation: TJ, tight junction.

was safer and less irritating than paeoniflorin. In addition, the toxicities of the different compounds to the cells were concentration dependent; the maximum doses of the various compounds in the cells were determined from the results, which guided the other experiments in this study.

The transport of puerarin in Calu-3 cell monolayers at different concentrations showed that the $P_{\text {app }}$ values (both $\mathrm{A} \rightarrow \mathrm{B}$ and $\mathrm{B} \rightarrow \mathrm{A}$ ) exhibited no significant difference, and the puerarin transport speed increased with an increase in concentration, suggesting that the dominant transport

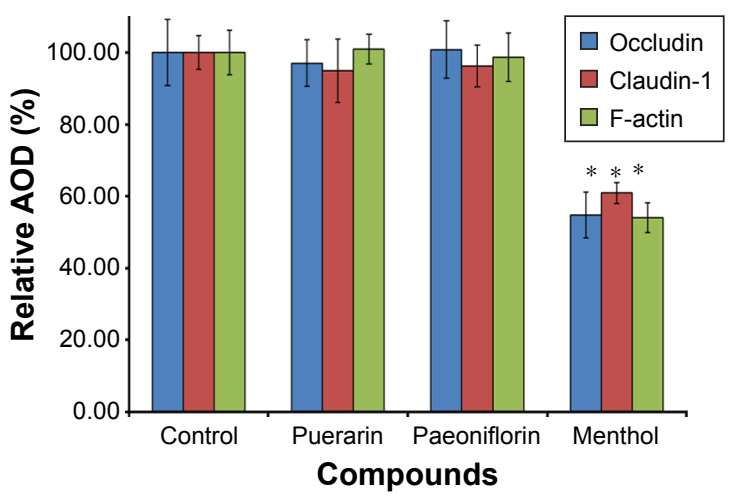

Figure 5 Changes to the relative AOD of occludin, claudin-I, and F-actin in Calu-3 cells after treatment with different compounds.

Notes: The relative AOD values for TJ proteins did not significantly differ after treatment with puerarin or paeoniflorin compared with the control group $(P>0.05)$, whereas the values of the menthol group decreased significantly $(P<0.05)$. Data shown as mean $\pm S D(n=3)$. $* P<0.05$ compared with the value in the control group.

Abbreviations: AOD, average optical density; T], tight junction; SD, standard deviation. mechanism of puerarin across the Calu-3 cell monolayer is the passive paracellular transport pathway. The ER values obtained for different puerarin concentrations were all $\sim$, and the transport of puerarin in both directions did not significantly differ; thus, the results in this study did not directly indicate that the transport of puerarin was related to the mucosal direction. It has been reported in the literature that the transport of puerarin in cell monolayers is a comprehensive process that mainly involves passive diffusion but may also involve active transportation mediated by P-glycoprotein (P-gp). ${ }^{35,36} \mathrm{P}$-gp is an important efflux pump involved in transport across the cell membrane that can transport compounds from inside the cell back into the extracellular fluid. It has also been noted that when the compounds are administered at a lower concentration, the effects of P-gp are more significant, whereas at higher concentrations, the simple diffusion rates of many P-gp substrates are much greater than the efflux. In the dosage range tested in this study, the results regarding transport indicated only the passive diffusion of puerarin. The assays evaluating the influence of drug compatibility on puerarin transport showed that paeoniflorin did not improve the permeability of puerarin. Some studies have reported that paeoniflorin is a P-gp substrate. ${ }^{37,38}$ In theory, when combined with paeoniflorin, the transport of puerarin should be improved due to competitive binding of the P-gp receptor by paeoniflorin. However, in 
our research, the $P_{\text {app }}$ values obtained for puerarin in combination with different concentrations of paeoniflorin did not increase significantly. This may be because the transport of puerarin in Calu-3 cells mainly occurs via passive diffusion. Therefore, in this complex prescription, paeoniflorin is used mainly as a medicinal component to enhance the therapeutic effect. In addition, menthol significantly facilitated puerarin transport, and the $P_{\text {app }}$ values of puerarin in the Calu-3 cell monolayers increased significantly with an increasing concentration of menthol. Many researchers have reported the use of menthol as a penetration enhancer. ${ }^{39,40}$ In previous in vivo studies, we have found that the brain targeting coefficient of puerarin combined with menthol and that the brain drug targeting index were significantly higher than those obtained with injections. ${ }^{34}$ Through studying the influence of menthol on the mucosal permeability of puerarin in the abdominal skin of Rana catesbeiana as an in vitro model, we also found that when the mass concentration of menthol was $5 \mathrm{mg} / \mathrm{mL}$ after compatibility with puerarin was assessed, the $P_{\text {app }}$ value was significantly higher than that obtained in the puerarin alone group. ${ }^{41}$ All these in vitro and in vivo results prove that menthol can enhance the permeation of puerarin in nasal administration and is beneficial for the transport of puerarin from the nose to the brain. Interestingly, when we assessed the ER values of puerarin transport with menthol, we observed that although puerarin in combination with menthol effectively permeated into the tissues, it also easily flowed out of the tissue. Nevertheless, the research in vivo still confirmed that puerarin reached the brain with sufficient availability. ${ }^{34}$ Therefore, we speculated that menthol promotes puerarin transport across the nasal mucosa and increases the chances of puerarin transport into the brain, but after penetrating the nasal mucosal epithelial cells, the mechanism of further transport could not be verified in that study and was necessary to study further.

There is very little information in the literature on the mechanism by which menthol enhances the permeability of the mucosal epithelial cells. Using a cell model of the nasal mucosa and the addition of menthol, we have described the reduction of TEER and the suppression of TJ proteins. Drug nasal absorption often involves paracellular transport. ${ }^{42,43}$ The regulation of paracellular transport across a monolayer involves multiple factors, in which the critical components are the degree of compactness and the physiological function between cells. TEER measurements were performed to evaluate the restrictiveness and to characterize the paracellular resistance of epithelial monolayers in vitro because the TEER value is affected by cell-substrate contact. If the distance between a cell and a substrate is small, the TEER value must be high. In this paper, the TEER value gradually decreased when menthol was added to the cells, whereas the values of the other groups were relatively stable during the test period. Thus, it was confirmed that menthol loosened the monolayer and weakened the nasal mucosa barrier to enhance puerarin paracellular transport. The mechanism by which menthol decreases the TEER values probably involves calcium influx and variations in the activity of intrinsic membrane proteins. ${ }^{44}$ The TJs surrounding epithelial cells also play a vital role in drug transport by tightly connecting neighboring cells and establishing a defined intercellular space. The TJs separate the apical domain from the basolateral cell surface domain, generating cell polarity and performing barrier and fence functions that restrict the paracellular transport of drugs. TJs consist of a series of integral membrane proteins, including claudins, occludin, zonula occludens, and actin..$^{45,46}$ In this study, representative TJ proteins were selected to study the influence of each compound on the TJ structure. Occludin, which is an $\sim 60 \mathrm{kDa}$ transmembrane protein with a short intracellular curve, two extracellular annuli, and N- and $\mathrm{C}$-termini in the cytoplasm, is regarded as the primary module in TJ strands that maintains epithelial polarity. ${ }^{47-49}$ Claudin-1, an 18-27 kDa transmembrane protein with two extracellular annuli, a short $\mathrm{N}$-terminus, and a C-terminus in the cytoplasmic domain, is also considered an important component of TJs and has even been referred to as the TJ backbone. ${ }^{50,51}$ Many recent studies have shown that claudin-1 is a critical component of TJs with cell adhesion activity that can directly affect the regulation of paracellular permeability and selectivity for solute size by interacting with itself to generate TJ strands. F-actin, one of the peripheral TJ membrane proteins, is involved in organelle movement, protoplasmic streaming, and intercellular junction regulation. ${ }^{45,52,53}$ Based on the experimental results of immunofluorescence testing and the semiquantitative analysis of the calculated AOD of these chosen TJ proteins, it was speculated that menthol had a disruptive effect on the configuration and integrity of TJs. These results were consistent with the TEER measurements and revealed that menthol suppressed the function of TJ proteins to improve the puerarin permeability across the nasal mucosa. The mechanism underlying the effects of menthol may be based on the phosphorylation of TJ components, activation of protein kinases, or depletion of calcium. ${ }^{54,55}$

Most drugs used in traditional Chinese medicine are extracted from well-known plants, and as such, their effects have been studied over their long history of use. In many cases, the safety and efficacy of these drugs have 
been established, but traditional dosages are unable to accommodate modern needs. By doing this research, we hope to provide information regarding the transport of puerarin and the influences of paeoniflorin and menthol on puerarin permeability at the cell level, thus confirming the rationality of using nasal drug delivery systems and the validity of the drug compatibility. However, transport from the nose to the brain is a complicated and comprehensive process. Therefore, this study is not a complete description of this type of transport, and further research is required.

\section{Conclusion}

We analyzed the cytotoxicity of compounds in Calu-3 cells, the bidirectional transport of puerarin across Calu-3 cell monolayers, and the influence of drug compatibility on mucosal permeation in vitro. The results confirmed that the transport of puerarin mainly occurred via passive diffusion; this transport was increased by menthol but not by paeoniflorin. Moreover, because menthol disrupted the TJ protein structure and weakened the barrier function of the epithelial cells, the use of menthol enhanced the mucosal permeation of puerarin. The experiments performed herein were conducted to better understand the fundamental pharmaceutical properties of puerarin in combination with paeoniflorin and menthol and to lay the foundation for deeper investigations into nasal administration and nosebrain pathways in vitro.

\section{Acknowledgments}

The authors thank Jing Han of the Beijing University of Chinese Medicine for her suggestions concerning immunofluorescence experiments and Zhen-Zhen Chen of Capital Medical University for his helpful advice for cell culture experiments. This project was supported by the National Natural Science Foundation of China (No 81473363) and the Self-Topic Fund of Beijing University of Chinese Medicine (No 2015-JYB-XS053).

\section{Disclosure}

The authors report no conflicts of interest in this work.

\section{References}

1. Hafner A, Škrinjar D, Filipovic-Grcic J. Nasal administration of medicines. Farmaceutski Glas. 2014;70(5):303-321.

2. Mistry A, Stolnik S, Illum L. Nanoparticles for direct nose-to-brain delivery of drugs. Int J Pharm. 2009;379(1):146-157.

3. Duchi S, Ovadia H, Touitou E. Nasal administration of drugs as a new non-invasive strategy for efficient treatment of multiple sclerosis. J Neuroimmunol. 2013;258(1-2):32-40.
4. Stockhorst U, Pietrowsky R. Olfactory perception, communication, and the nose-to-brain pathway. Physiol Behav. 2004;83(1):3-11.

5. Sarkar A. Drug metabolism in the nasal mucosa. Pharm Res. 1992; 9(1):1-9.

6. Okuyama S. The first attempt at radioisotopic evaluation of the integrity of the nose-brain barrier. Life Sci. 1997;60(21):1881-1884.

7. Casettari L, Illum L. Chitosan in nasal delivery systems for therapeutic drugs. J Control Release. 2014;190:189-200.

8. Kürti L, Gáspár R, Márki Á, et al. In vitro and in vivo characterization of meloxicam nanoparticles designed for nasal administration. Eur J Pharm Sci. 2013;50(1):86-92.

9. Agu RU, Jorissen M, Willems T, Augustijns P, Kinget R, Verbeke N. Invitro nasal drug delivery studies: comparison of derivatised, fibrillar and polymerised collagen matrix-based human nasal primary culture systems for nasal drug delivery studies. J Pharm Pharmacol. 2001;53(11): $1447-1456$.

10. Hussainx AA, Hirai S, Bawarshi R. Nasal absorption of natural contraceptive steroids in rats - progesterone absorption. J Pharm Sci. 1981;70(4):466-467.

11. Steele VE, Arnold JT. Isolation and long-term culture of rat, rabbit, and human nasal turbinate epithelial cells. In Vitro Cell Dev Biol. 1985;21(12): 681-687.

12. Merkle HP, Ditzinger G, Lang SR, Peter H, Schmidt MC. In vitro cell models to study nasal mucosal permeability and metabolism. Adv Drug Deliv Rev. 1998;29(1-2):51-79.

13. Wengst A, Reichl S. RPMI 2650 epithelial model and three-dimensional reconstructed human nasal mucosa as in vitro models for nasal permeation studies. Eur J Pharm Biopharm. 2010;74(2):290-297.

14. Vllasaliu D, Fowler R, Garnett M, Eaton M, Stolnik S. Barrier characteristics of epithelial cultures modelling the airway and intestinal mucosa: a comparison. Biochem Biophys Res Commun. 2011;415(4):579-585.

15. Tayebati SK, Nwankwo IE, Amenta F. Intranasal drug delivery to the central nervous system: present status and future outlook. Curr Pharml Des. 2013;19(3):510-526.

16. Jerey JL, Robert GT. Intranasal Drug Delivery to the Brain. Drug Delivery to the Brain. New York, NY: Springer; 2014:401-431.

17. Qin $\mathrm{T}$, Yin $Y$, Wang $X$, et al. Whole inactivated avian Influenza H9N2 viruses induce nasal submucosal dendritic cells to sample luminal viruses via transepithelial dendrites and trigger subsequent DC maturation. Vaccine. 2015;33(11):1382-1392.

18. Miyazawa M, Sakano K, Nakamura S, Kosaka H. Antimutagenic activity of isoflavone from Pueraria lobata. J Agric Food Chem. 2001;49(1): 336-341.

19. Wong KH, Li GQ, Li KM, Razmovski-Naumovski V, Chan K. Kudzu root: traditional uses and potential medicinal benefits in diabetes and cardiovascular diseases. J Ethnopharmacol. 2011;134(3):584-607.

20. Zhang H, Zhang L, Zhang Q, et al. Puerarin: a novel antagonist to inward rectifier potassium channel (I K1). Mol Cell Biochem. 2011; 352(1-2):117-123.

21. Liu Y, Xue Q, Li X, et al. Amelioration of stroke-induced neurological deficiency by lyophilized powder of Catapol and Puerarin. Int J Biol Sci. 2014;10(4):448-456.

22. Yiguo Z, Mei X, Zhinneg Y, et al. Analysis of puerarin and chemical compositions changes in kudzu root during growth period. J Chem. 2014;2014:1-6.

23. Zengxiang Q. [Pharmacological action and application of Gegentang]. Chinese Traditional Patent Material. 1996;18(4):43-44. Chinese.

24. Jian Z, Min Z. [Gegentang treated 36 post-stroke depression patients]. J Jilin Univ Med Ed. 2009;3(9):569. Chinese.

25. Jiai Y, Yue D, Shupang A. [Pharmacological and clinical researches of Gegentang]. Arch Tradit Chin Med. 2007;25(6):1275-1278.

26. Mikage M, Ono N. Herbological study of red peony and white peony used in Chinese medicine. Kampo Med. 2009;60(4):419-428.

27. Zhang Y, Li H, Huang M, et al. Paeoniflorin, a monoterpene glycoside, protects the brain from cerebral ischemic injury via inhibition of apoptosis. Am J Chin Med. 2015;43(3):543-557. 
28. Xiao L, Wang YZ, Liu J, Luo XT, Ye Y, Zhu XZ. Effects of paeoniflorin on the cerebral infarction, behavioral and cognitive impairments at the chronic stage of transient middle cerebral artery occlusion in rats. Life Sci. 2005;78(4):413-420.

29. Gelal A, Jacob P, Yu L, Benowitz NL. Disposition kinetics and effects of menthol. Clin Pharmacol Ther. 1999;66(2):128-135.

30. Plevkova J, Kollarik M, Poliacek I, et al. The role of trigeminal nasal TRPM8-expressing afferent neurons in the antitussive effects of menthol. J Appl Physiol. 2013;115(2):268-274.

31. Amato A, Liotta R, Mulè F. Effects of menthol on circular smooth muscle of human colon: analysis of the mechanism of action. Eur J Pharmacol. 2014;740:295-301.

32. Chen X, Du S, Lu Y, et al. [Study on pharmacokinetics of puerarin in rats following different methods of administration of Tongqiaosanyu prescription]. Zhongguo Zhong Yao ZaZhi. 2011;36(17):2347-2349. Chinese.

33. Xiaolan C, Shouying D, Yang L, et al. [Study on pharmacokinetics of puerarin in rats by following different methods of administration of puerariae extract]. Chin J Tradit Chin Med Pharmacy. 2011 27(10):2408-2411. Chinese.

34. Xiaolan C, Yang L, Shouying D, et al. Study on the comparison the plasma pharmacokinetics with the brain pharmacokinetics based on the different administration routes of TongqiaoSanyu formula. China J Tradit Chin Med Pharmacy. 2012;29(8):2668-2672.

35. Liang XL, Zhao LJ, Liao ZG, et al. Transport properties of puerarin and effect of radix Angelicae dahuricae extract on the transport of puerarin in Caco-2 cell model. J Ethnopharmacol. 2012;144(3):677-682.

36. Liang XL, Zhang J, Zhao GW, et al. Mechanisms of improvement of intestinal transport of baicalin and puerarin by extracts of radix Angelicae dahuricae. Phytother Res. 2015;29(2):220-227.

37. Liu L, Zhao X, Zhu D, Cheng Y, Qu H. Simultaneous LC-MS/MS determination of danshensu and paeoniflorin for permeability studies in Caco-2 intestinal absorption model. Chem Res Chin Univ. 2008; 24(4):420-426.

38. Liang XL, Zhu ML, Zhao GW, et al. [Effect of furan coumarins from Angelica dahuricae radix on intestinal transport absorption of puerarin, paeoniflorin, and vincristine]. Tradit Herbal Drug. 2015;46(7): 1007-1011. Chinese.

39. Xu X, Yu N, Bai Z, et al. Effect of menthol on ocular drug delivery. Graefes Arch Clin Exp Ophthalmol. 2011;249(10):1503-1510.

40. Huang L, Bai J, Yang H, Liu J, Cui H. Combined use of borneol or menthol with labrasol promotes penetration of baicalin through rabbit cornea in vitro. Pak J Pharm Sci. 2015;28(1):1-7.
41. Pengyue L, Yang L, Shouying D, Xiaolan C. [Study on the effect of menthol on mucosal permeability of Puerarin in vitro]. Chin Pharmacy. 2012;23(47):4425-4427. Chinese.

42. Genter MB, Krishan M, Augustine LM, Cherrington NJ. Drug transporter expression and localization in rat nasal respiratory and olfactory mucosa and olfactory bulb. Drug Metab Dispos. 2010;38(10): 1644-1647.

43. Huang Y, Donovan MD. Large molecule and particulate uptake in the nasal cavity: the effect of size on nasal absorption. Adv Drug Deliv Rev. 1998;29(1-2):147-155.

44. Turner JR, Buschmann MM, Romero-Calvo I, Sailer A, Shen L. The role of molecular remodeling in differential regulation of tight junction permeability. Semin Cell Dev Biol. 2014;36:204-212.

45. Tsukita S, Furuse M, Itoh M. Multifunctional strands in tight junctions. Nat Rev Mol Cell Biol. 2001;2(4):285-293.

46. Sawada N, Murata M, Kikuchi K, et al. Tight junctions and human diseases. Med Electron Microsc. 2003;36(3):147-156.

47. Balda MS, Whitney JA, Flores C, González S, Cereijido M, Matter K. Functional dissociation of paracellular permeability and transepithelial electrical resistance and disruption of the apical-basolateral intramembrane diffusion barrier by expression of a mutant tight junction membrane protein. J Cell Biol. 1996;134(4):1031-1049.

48. Furuse M, Hirase T, Itoh M, et al. Occludin: a novel integral membrane protein localizing at tight junctions. J Cell Biol. 1993;123(6 pt 2) 1777-1788.

49. Tsukamoto T, Nigam SK. Role of tyrosine phosphorylation in the reassembly of occludin and other tight junction proteins. Am J Physiol. 1999;276(5 pt 2):F737-F750.

50. Furuse M, Fujita K, Hiiragi T, Fujimoto K, Tsukita S. Claudin-1 and -2: novel integral membrane proteins localizing at tight junctions with no sequence similarity to occludin. J Cell Biol. 1998;141(7):1539-1550.

51. Furuse M, Sasaki H, Fujimoto K, Tsukita S. A single gene product, claudin-1 or -2 , reconstitutes tight junction strands and recruits occludin in fibroblasts. J Cell Biol. 1998;143(2):391-401.

52. Schneeberger EE, Lynch RD. The tight junction: a multifunctional complex. Am J Physiol Cell Physiol. 2004;286(6):C1213-C1228.

53. Hall A. Rho GTpases and the actin cytoskeleton. Science. 1998;279(5350):509-514.

54. Velarde G, Ait-Aissa S, Gillet C, et al. Use of transepithelial electrical resistance in the study of pentachlorophenol toxicity. Toxicol In Vitro. 1999;13(4-5):723-727.

55. Hirsch M, Noske W. The tight junction: structure and function. Micron $1993 ; 24(3): 325-352$.
Drug Design, Development and Therapy

\section{Publish your work in this journal}

Drug Design, Development and Therapy is an international, peerreviewed open-access journal that spans the spectrum of drug design and development through to clinical applications. Clinical outcomes, patient safety, and programs for the development and effective, safe, and sustained use of medicines are a feature of the journal, which

\section{Dovepress}

has also been accepted for indexing on PubMed Central. The manuscript management system is completely online and includes a very quick and fair peer-review system, which is all easy to use. Visit http://www.dovepress.com/testimonials.php to read real quotes from published authors. 